\title{
Interpersonal attraction as a joint function of primacy and recency effects*
}

\author{
PAUL A. BELL \\ Purdue University, West Lafayette, Indiana 47907
}

\begin{abstract}
Empirical primacy-recency inconsistencies between Byrne's reinforcement affect model of attraction and Anderson's information integration model of personality impression formation were explored. Thirty-six male and female Ss gave attraction responses to sequences of blocks of similar and dissimilar attitudes arranged in increasing or decreasing proportions of similarity and increasing or decreasing levels of topic importance. Results generally supported the hypothesis that attraction is a joint function of the proportion of similar attitudes within each block (recency) and cumulative proportion of similar attitudes before each response (primacy). This joint occurrence of primacy and recency effects was interpreted in terms of an affect neutralization hypothesis.
\end{abstract}

Considerable attention has been focused in recent years on research involving primacy and recency effects in the serial development of interpersonal attraction and the serial formation of personality impressions. In the typical impression formation experiment, positive and negative adjectives describing a hypothetical stranger usually has the most influence on the ensuing personality impression (Anderson, 1965; Asch, 1946).

The typical interpersonal attraction experiment, on the other hand, employs as the stimulus material a set of attitudes held by a bogus stranger and constructed in such a way as to agree or disagree with a systematically determined proportion of the S's attitudes (see, e.g., Byrne \& Nelson, 1965). In contrast to personality impression formation tasks, a single attraction response at the end of a series of attitudinal stimuli yields neither a primacy nor a recency effect. Rather, the attraction response is apparently a weighted average of the proportion of similar attitudes without regard to the serial position of the similar and dissimilar attitudes (Byrne \& London, 1966).

In an attempt to resolve the primacy-recency discrepancies between attraction and information integration paradigms, Byrne, Lamberth, Palmer, and London (1969) presented a series of 24 similar and dissimilar attitudes such that the proportion of similar attitudes in six blocks of four attitudes each either ascended from .00 to 1.00 or descended from 1.00 to .00 , with the overall proportion of similarity being .50 after the last block. If one attraction response was obtained only at the end of the last block of attitudes (final responding), no primacy or recency effects were found, thus replicating the Byrne and London (1966) results. However, if responses were made after each of

* Data for this study were collected as a part of the author's Master's thesis at Trinity University in San Antonio, Texas. The author expresses his appreciation to the members of his thesis committee, L. R. Stanley, V. A. Benignus, J. W. Worsham, and Louie Miller III for their assistance in the development of this research. Requests for reprints should be sent to Paul Bell, Department of Psychological Sciences, Purdue University, West Lafayette, Indiana 47907. the six blocks of attitudes (intermediate responding) or after each of the 24 individual attitudes (continuous responding), strong recency effects were found. That is, the intermediate attraction response after each block was solely determined by the proportion of similar attitudes within that block, and similar and dissimilar attitudes preceding that block had no effect on attraction. Hoffman, Kaplan, and Stewart (1974) reported similar recency effects for intermediate responding, and such recency effects have also been reported in personality impression formation tasks that involved interpolated responses (e.g., Anderson, 1968; Hendrick \& Constantini, 1970; Stewart, 1965).

Interestingly, Byrne et al (1969) found the recency effect for intermediate responding to be stronger than the (nonexistent) recency effect for final responding but weaker than the recency effect for continuous responding. Rather than either primacy or recency operating alone, there appeared to be a gradual shift along a continuum from primacy to recency depending on the frequency of interpolated responses. One implication of this gradual shift is that with intermediate responding primacy and recency effects may be operating simultaneously, so that attraction may be a joint function of agreement on initial attitudes (primacy) and agreement on attitudes encountered since the last attraction response (recency). If this is indeed the case, one would expect blocks of attitudes progressing from 1.00 similarity to .00 similarity to elicit higher attraction responses than equivalent blocks progressing from .00 to 1.00 similarity because of the initial agreement in the former sequence and the initial disagreement in the latter. A reexamination of the Byrne et al (1969) data, however, shows no such primacy effects $(\mathrm{p}>.05){ }^{1}$

Nevertheless, joint primacy and recency effects are at least theoretically possible within the context of the Byrne-Clore model of interpersonal attraction (Byrne, 1971). According to this reinforcement affect model, the weight of a particular similar or dissimilar attitude 
(or other reinforcing stimulus) in determining the attraction response is dependent on the magnitude of the reinforcing properties embodied in the particular stimulus. For attitudinal stimuli this magnitude of reinforcement is operationalized in terms of the topic importance of the attitudes (see, e.g., Clore \& Baldridge, 1968). Primacy-recency effects might also be interpreted as differences in weight of the attitudinal stimuli at tributable solely to the serial position of the attitude items (see also Anderson, 1965, 1968). Thus, the weight of a given attitudinal stimulus is theoretically determined by both the topic importance of the attitude and by the serial position of the attitude. Since the topic importance of the attitudes in the Byrne et al (1969) serial study was randomized within blocks of attitudes (Byrne, personal communication), it is impossible to separate adequately the topic importance and serial position weighting factors. The present study is designed to allow experimental control over botis topic importance and serial position of similar and dissimilar attitudes so that the potential joint occurrence of primacy and recency effects can be studied. Specifically, it is hypothesized that intermediate attraction responses after blocks of similar and dissimilar attitudes will be primarily dependent on the proportion of similar attitudes within the preceding block (recency) and that attraction responses for an initially agreeing series of attitudes will be higher than attraction responses for a comparably weighted (by means of topic importance) series of initially disagreeing attitudes (primacy).

\section{METHOD}

\section{Subjects}

Ss were 19 male and 17 female volunteers from St. Philips College in San Antonio, Texas.

\section{Procedure}

On the first day of the experiment, Ss completed a 100-item attitude survey consisting of bipolar six-point items covering such topics as political parties, fraternities, and science fiction. Ss also rated the topic importance of each item on a five-point scale (unimportant-important). A 20-item attitude survey, one item per page, was then prepared by the $E$ for each $S$ so that similar and dissimilar attitudes were systematically varied. Similar attitudes were defined as exact agreement with the S, and dissimilar attitudes were defined as three positions away on the opposite side of the neutral point. For half of these bogus surveys (DS), successive blocks of four attitude items each increased in proportion of similarity from .00 to 1.00 in increments of .25 , so that the following pattern of similar and dissimilar attitudes appeared: DDDD DSDD SDSD SSSD SSSS. For the other half of the bogus surveys (SD), successive blocks decreased in proportion of similarity from 1.00 to .00 : SSSS SDSS DSDS DDDS DDDD. In addition, S-rated topic importance was made homogeneous within each block and across blocks either increased from unimportant to important (u-i) or decreased from important to unimportant (i-u). Thus, there were four groups of Ss based on the arrangement of the blocks of attitudes: similar-important to dissimilar-unimportant (SiDu), similar-unimportant to dissimilar-important (SuDi), dissimilar-important to similar-unimportant (DiSu), and dissimilar-unimportant to similar-important (DuSi). In addition to the pages of individual attitudes, one page consisting of two response scales was inserted after each block of four attitude items. The two response scales consisted of a 13-point attraction scale (dislike-like) and a buffer scale asking for a judgment of the bogus stranger's educational level (uneducated-educated).

On the second day of the experiment, a bogus attitude survey was given to each $\mathrm{S}$, who was told this survey was completed by another $S$ in a similar experiment. Ss were instructed to observe the "stranger' $s$ " attitudes and to make the requested judgments about this person as each page of response scales was encountered. Because several $S s$ failed to complete the experiment, unequal numbers of $S s$ resulted in each experimental group (SiDu, 8; SuDi, 10; DiSu, 9; DuSi, 9), although sex and age of $S s$ were randomized across the four groups.

\section{RESULTS}

As in the Byrne et al (1969) study, a constant of 1.00 was added to all attraction scores to give the data a range of 2 to 14 , which is the more conventional range used in Byrne's 13-point Interpersonal Judgment Scale index of attraction (Byrne, 1971). Mean attraction responses for each block of attitudes are presented in Table 1 for each of the four experimental groups. Inspection of these means for the last block suggests that, as anticipated, Ss receiving terminal agreement gave higher attraction responses than Ss receiving terminal disagreement and that this difference was greatest when the last block of attitudes concerned important topics (i.e., Groups DuSi and SuDi). An analysis of variance performed on the data for the last block indicated significance for both the main effect of similarity $(\mathrm{F}=8.82, \mathrm{df}=1 / 32, \mathrm{p}<.006)$ and the interaction between that factor and topic importance $(\mathrm{F}=4.04, \mathrm{df}=1 / 32, \mathrm{p}<.05)$. Thus, even though Ss had received $50 \%$ agreement overall after the last block of attitudes, the order of presentation of agreeing and disagreeing attitudes influenced the final attraction response, especially when the last block contained important attitudes.

Further examination of Table 1 suggests that $S s$ responded strongly to the increasing or decreasing proportion of similarity across blocks. Indeed, an analysis of variance on the data for all five blocks indicated a strong interaction of Blocks by Order of Proportion of Similarity $(F=18.61, d f=4 / 128$, $\mathrm{p}<.001)$. Thus, in accord with the results of Byrne et al (1969) and Hoffman et al (1974), proportion of similarity within the most recent block of attitudes

Table 1

Mean Attraction Responses for Four Experimental Groups

\begin{tabular}{lrrrrr} 
& \multicolumn{5}{c}{ Blocks of Four Attitudes } \\
\cline { 2 - 6 } Group* & \multicolumn{1}{c}{1} & \multicolumn{1}{c}{2} & \multicolumn{1}{c}{3} & \multicolumn{1}{c}{4} & \multicolumn{1}{c}{5} \\
\hline DuSi & 8.78 & 8.78 & 10.00 & 10.89 & 11.44 \\
DiSu & 5.89 & 5.89 & 8.33 & 8.56 & 10.22 \\
SuDi & 11.30 & 11.90 & 10.80 & 9.60 & 6.40 \\
SiDu & 11.00 & 9.63 & 10.50 & 9.75 & 9.25 \\
\hline
\end{tabular}

${ }^{*} D=$ dissimilar, $S=$ similar, $u=$ unimportant, $i=$ important . 


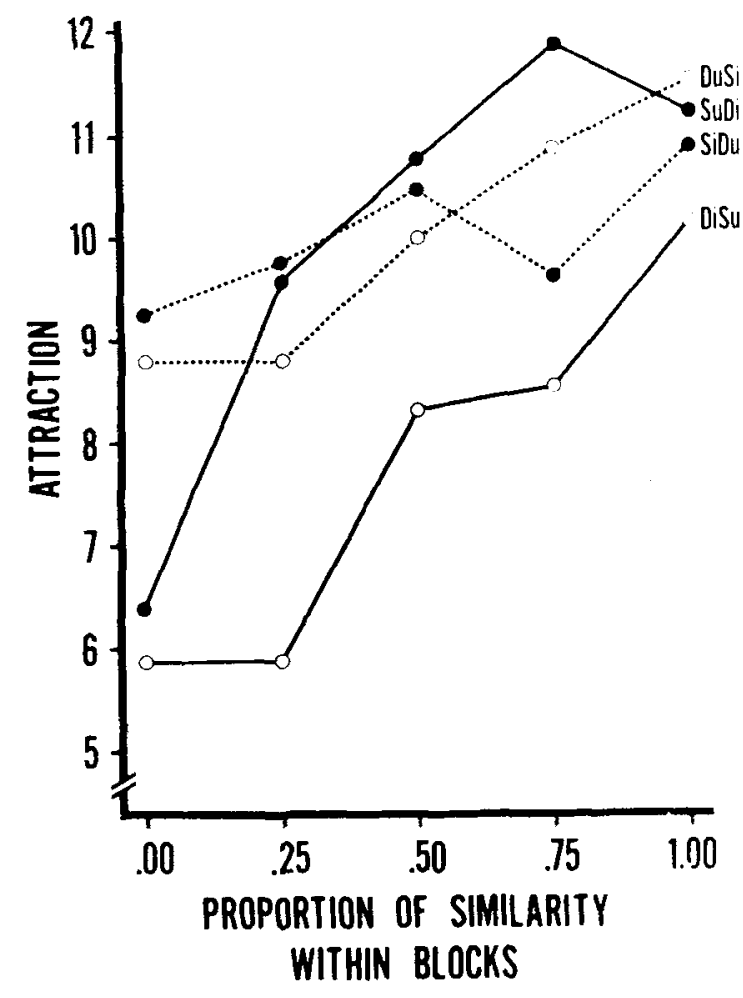

Fig. 1. Attraction as a function of proportion of similarity within blocks for four sequences of attitude presentation: dissimilar-unimportant to similar-important (DuSi), similar-unimportant to dissimilar-important (SuDi), similar-important to dissimilar-unimportant (SiDu), and dissimilar-important to similar-unimportant (DiSu).

appears to be a principal determinant of sequential attraction responses. Whether or not proportion of similarity in preceding blocks also influences these responses (i.e., primacy effect) cannot readily be determined from analyzing the data as formatted in Table 1, since, with "blocks" as a variable, proportion of similarity in previous blocks is confounded with topic importance. A more appropriate analysis would use proportion of similarity within blocks as a variable, as indicated in Fig. 1. In this way, groups whici have had the same degrees of topic importance paired with each proportion of similarity (e.g., SuDi vs DiSu, SiDu vs DuSi) can be compared, with the only difference between compared groups being the order of presentation of agreement. For example, Groups SuDi and DiSu both had unimportant topics paired with 1.00 similarity blocks and important topics paired with .00 similarity blocks. However, Group SuDi began with agreeing attitudes and Group DiSu began with disagreeing attitudes. If agreement in preceding blocks influences attraction responses in subsequent blocks, one would expect the initial agreement in Group SuDi to produce higher attraction responses than the initial disagreement in Group DiSu. Indeed, examination of Fig. 1 suggests that this order of agreement difference produced consistently higher attraction responses in
Group SuDi than in Group DiSu. Simple effects analyses comparing these two groups revealed significant effects for groups $(F=4.77, \mathrm{df}=1 / 32, \mathrm{p}<.05)$, proportion of similarity $(F=18.40, d f=4 / 128, p<.001)$, and the interaction of these two factors $(F=2.45, d f=4 / 128$, $p<.05$ ). Analogous simple effects analyses comparing Groups DuSi and SiDu (see Fig. 1), both of which had important topics paired with 1.00 blocks and unimportant topics paired with .00 blocks, yielded only a significant effect for proportion of similarity $(F=3.53, \mathrm{df}=4 / 128, \mathrm{p}<.01$, all other $\mathrm{Fs}<1.00)$. Thus, at least in the SuDi and DiSu groups, both the proportion of similarity in the most recent block and the proportion of agreement in previous blocks appeared to influence Ss' at traction responses.

In order to gain further insight into this apparently joint influence of similarity in both most recent and preceding blocks of attitudes, the attraction data from all four experimental groups were combined in a multiple regression analysis having as predictors proportion of similarity within blocks (recency), accumulated proportion of similarity before each response $^{2}$ (primacy), and topic importance (magnitude of reinforcement). Results indicated that recency effects accounted for $15 \%$ of the variability, primacy effects $3 \%$, and magnitude of reinforcement $2 \%$.

\section{DISCUSSION}

Consistent with similar findings by Byrne et al (1969) and Hoffman et al (1974), the results of the present study indicate strong recency effects for attraction responses interpolated within a series of similar and dissimilar attitudes. In addition, these results suggest the presence of simultaneous primacy effects in that such attraction responses are also influenced by agreement in earlier blocks of attitudes. However, these primacy effects appear difficult to detect unless very large or very small magnitudes or reinforcement are associated with initially or teminally low proportions of similar attitudes. Even when detectable, however, these primacy effects account for relatively little variability in attraction responses when compared with recency effects.

The results also suggest that high magnitudes of negative reinforcement (i.e., disagreement on important topics) facilitate the occurrence of these primacy effects, as was demonstrated in the DiSu and SuDi groups. However, since no clear primacy effects occurred for groups having high magnitudes of positive reinforcement (i.e., DuSi and $\mathrm{SiDu}$ ), the relationship between weighting due to serial position and weighting due to topic importance appears to be relatively complex. Indeed, since the Byrne et al (1969) data show no primacy effects when topic importance is randomized across blocks, it seems very probable that the primacy effects in the present study are at least somewhat dependent on 
the unique combinations of topic importance and proportion of similarity employed. Thus, it would not seem to be inconsistent either with previous formulations of weighting systems (see, e.g., Clore \& Baldridge, 1968) or with the present results to suggest an interactive relationship between topic importance and serial position in determining the weight of an individual attitude. Specifying the exact nature of this relationship must await more elaborate combinations of the serial position and magnitude of reinforcement parameters.

Of more important interest for the present are the theoretical implications of the present results. Several cognitive explanations, such as a change in meaning hypothesis (Asch, 1946), attention decrement (Anderson, 1965), and discounting of later stimuli that are inconsistent with earlier stimuli (Anderson \& Jacobson, 1965), have been proposed for primacy-recency effects in personality impression formation tasks. However, using these explanations to interpret results from the affect-oriented procedures of the present study involves a peculiar though interesting hybridization of cognitive and affective theoretical notions. Although such hybridizations may or may not be desirable (Byrne, Clore, Griffitt, Lamberth, \& Mitchell, 1973; Kaplan \& Anderson, 1973), in the interest of simplicity and theoretical purity an alternative (and possibly more parsimonious) explanation of the present results can be offered within the context of Byrne's reinforcement affect paradigm (Byrne, 1971).

According to Byrne's model, attraction toward a person is a function of the affect previously associated with that person. This affect may originate from any stimuli with reinforcing properties, such as similar and dissimilar attitudes. Moreover, independently inducing positive or negative affect, through the use of humorous or depressing films (Bleda, Bell, \& Byrne, 1973; Gouaux, 1971), for example, can independently raise or lower the affect associated with the stimulus person and can, thus, independently raise or lower attraction. Byrne et al (1969) suggest that giving an attraction response to the previous block of attitudes somehow "neutralizes" the affect associated with those attitudes, so that only the affect associated with the next block of attitudes influences the next attraction response (see also, Gouaux, 1972). Stronger affect, such as that associated with large magnitudes of all negative (.00 similarity) reinforcements, would seem much more difficult to neutralize with a single attraction response. The leftover nonneutralized affect from such a block of attitudes would then function as a source of affect independent of the affect associated with the next block of attitudes. Thus, the "induced" affect remaining from the previous block of attitudes would account for primacy effects, and the affect associated with the subsequent block of attitudes would account for simultaneous recency effects. Perhaps not by coincidence, the relative amounts of variability in attraction responses accounted for by primacy and recency effects in the present study correspond quite well with the relative amounts of variability accounted for by independently induced and reinforcement-associated affect in induced affect studies (e.g., Bleda et al, 1973). This affective explanation of joint primacy and recency effects is thus not only relatively parsimonious but also entirely consistent with Byrne's reinforcement-affect model of interpersonal attraction. More direct measures of affect in future research involving interpolated attraction responses should be very helpful in further assessing the viability of this affective interpretation of primacy-recency effects.

\section{REFERENCES}

Anderson, N. H. Primacy effects in personality impression formation using a generalized order effect paradigm. Journal of Personality \& Social Psychology, 1965, 2, 1-9.

Anderson, N. H. Application of a linear-serial model to a personality-impression task using serial presentation. Journal of Personality \& Social Psychology, 1968, 10, 354-362.

Anderson, N. H., \& Jacobson, A. Effect of stimulus inconsistency and discounting instructions in personality impression formation. Journal of Personality \& Social Psychology, 1965, 2, 531-539.

Asch, S. E. Forming impressions of personality. Journal of Abnormal \& Social Psychology, 1946, 41, 258-290.

Bleda, P. R., Bell, P. A., \& Byrne, D. Prior induced affect and sex differences in attraction. Memory \& Cognition, 1973, 1, 435-438.

Byrne, D. The attraction paradigm. New York: Academic Press, 1971 .

Byrne, D., Clore, G. L., Griffitt, W., Lamberth, I., \& Mitchell, H. E. When research paradigms converge: Confrontation or integration? Journal of Personality \& Social Psychology, $1973,28,313-320$.

Byrne, D., Lamberth, J., Palmer, J .. \& London, O. Sequential effects as a function of explicit and implicit interpolated attraction responses. Journal of Personality \& Social Psychology, 1969, 13, 70-78.

Byrne, D., \& London, $O$. Primacy-recency and the sequential presentation of attitudinal stimuli. Psychonomic Science, $1966,4,193-194$.

Byrne, D., \& Nelson, D. Attraction as a linear function of proportion of positive reinforcements. Journal of Personality \& Social Psychology, 1965, 1, 659-663.

Clore, G. L., \& Baldridge, B. Interpersonal attraction: The role of agreement and topic interest. Journal of Personality \& Social Psychology, 1968, 9, 340-346.

Gouaux, C. Induced af fective states and interpersonal attraction. Journal of Personality \& Social Psychology, 1971, 70, 37-43.

Gouaux C. Increased attraction to a stranger through neutralization of negative interpersonal affect by repeated evaluation. Psychonomic Science, 1972, 27, 101-102.

Hendrick, C., \& Constantini, A. F. Effects of varying trait inconsistency and response requirements on the primacy effect in impression formation. Journal of Personality \& Social Psychology, 1970, 15, 158-164.

Hoffman, J., Kaplan, M. F., \& Stewart, M. Order effects in attraction formation. Paper presented at the meeting of the Midwestern Psychological Association, Chicago, May 1974.

Kaplan, M. F., \& Anderson, N. H. Information integration theory and reinforcement theory as approaches to interpersonal attraction. Journal of Personality \& Social Psychology, 1973, 28, 301-312.

Stewart, R. H. Effect of continuous responding on the order effect in personality impression formation. Journal of Personality \& Social Psychology, 1965, 2, 161-165.

\section{NOTES}

1. Appreciation is expressed to Donn Byrne for providing access to his data and for his helpful comments and suggestions. 2. The same $3 \%$ variability is accounted for if primacy is operationally defined as the proportion of similarity in the first block of the sequence or as the proportion of similarity in the preceding block. No variability is accounted for by sex, age, or interactions.

(Received for publication April 5, 1974 revision accepted July $8,1974$. 